\section{Out of the kitchen}

Designer microwave ovens that can heat reactants in record time are heralding a quiet revolution in chemical synthesis. David Adam feels the heat.

$\mathrm{T}$ hey may still struggle to turn out a decent baked potato, but the twin virtues of speed and convenience have ensured that there is barely a modern kitchen without a microwave oven. And the same could soon be true of chemistry laboratories. A new generation of devices designed specifically for chemical synthesis is driving fresh interest in the power of microwaves among industrial and academic chemists alike.

As in the kitchen, speed is the strongest selling point for the devices. Microwaves heat reactants much more quickly than conventional means, so syntheses that usually take hours can be done in just a few minutes. Other reactions can be made more selective, producing more of a desired molecule and less contaminating by-products. "In 10-15

years, we will see a microwave reactor in every academic and industrial labora-

tory," predicts Oliver Kappe, who works on microwave synthesis at Graz University in Austria. "They will be the Bunsen burners of the twentyfirst century."

Microwaves have been used sporadically in scientific research

Hotting up: Oliver Kappe believes the demand for microwave devices in chemistry will grow.

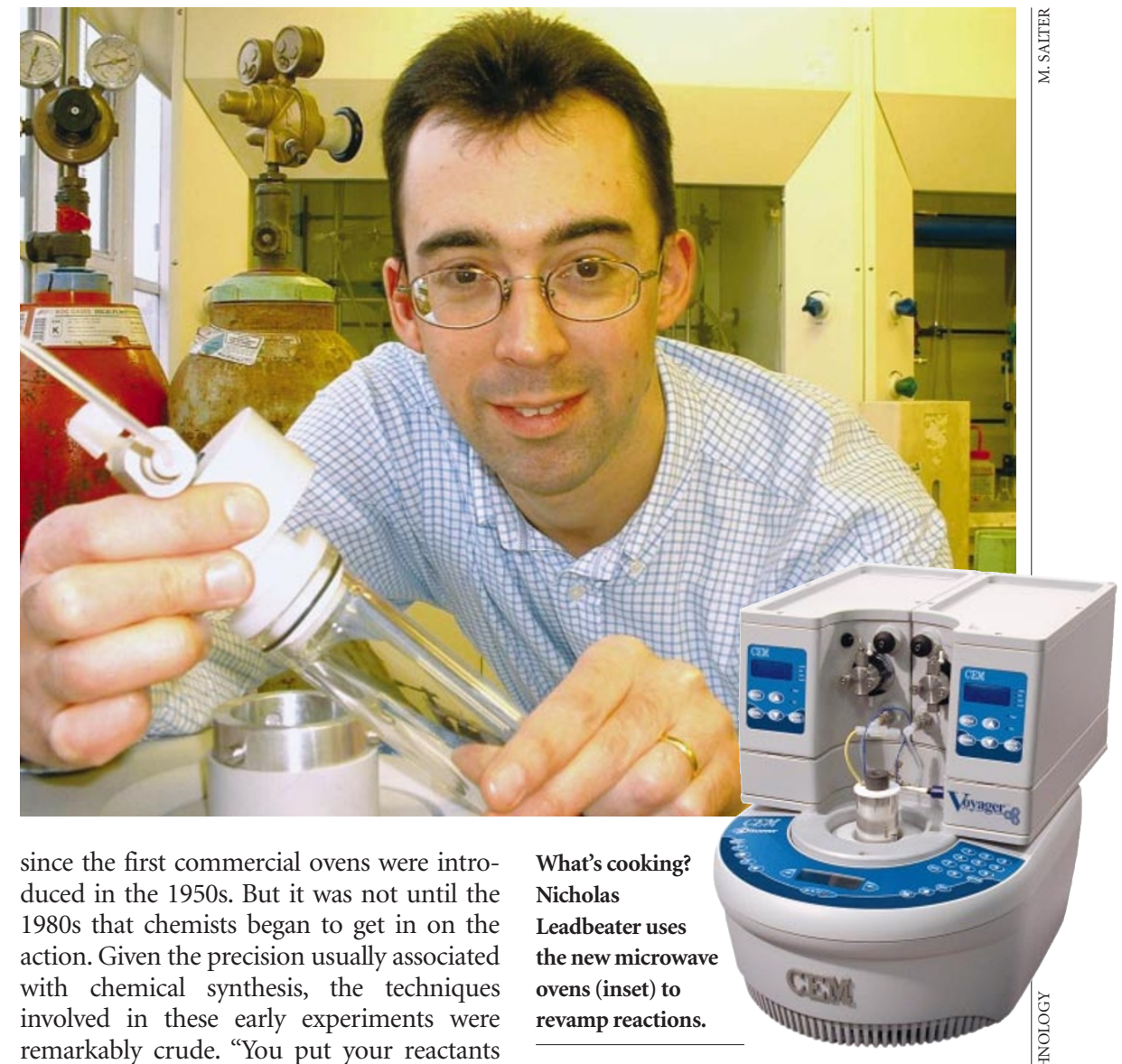
remarkably crude. "You put your reactants in a sealed vessel inside a conventional domestic oven, bedded it down in sand and blasted it with 'defrost', 'cook' or whatever,' recalls Nicholas Leadbeater, an organic chemist at King's College London.

Accidents were common. "Everyone who has done chemistry in a domestic microwave oven has had some kind of mishap," Leadbeater says. "Depending on what you were doing, either the vessel exploded inside the oven cavity or you just blew the door off." Those chemists who braved the risks saw interesting results - usually a phenomenal increase in reaction rate. But with no way to measure the temperature and pressure inside the oven, and frustrating variability between the results obtained using different models, experimental findings could rarely be reproduced reliably.

\section{Hot, hot, hot}

Things began to get more precise two or three years ago, when companies making specialist microwave equipment for applications in the food industry - such as breaking down fats for food-quality testing - realized that synthetic chemists would pay good money for more reliable machines. Chemists working in the pharmaceutical industry were the first to benefit, but since then a growing number of academic labs - including those of Kappe and Leadbeater - have also invested in the new tabletop devices.

The ovens allow accurate control of temperature and pressure, and include design features to contain exploding reactants "we still have about one explosion a week," Leadbeater admits. With these microwave reactors, synthetic chemists are now revisiting notoriously slow or temperamental reactions.

Leadbeater's group, for example, has reworked an important synthesis called the Suzuki reaction, which forges a link between two benzene rings to make 'biaryl' molecules. These organic structures are found in a wide range of compounds including pharmaceuticals, polymers and liquid crystals. Shifting the synthesis into a microwave reactor removes the need for an expensive palladium catalyst, making the process cheaper and less prone to contamination with traces of palladium ${ }^{1}$.

Microwave heating can also steer reactions towards desired products. Using conventional heat sources, for instance, the reaction between naphthalene and sulphuric acid produces an equal mixture of two possible isomeric forms of a molecule that is used in the manufacture of dyes and other industrially important chemicals. But the increased rate of heating obtained using microwaves means that the reaction can be driven to give one or other of the products ${ }^{2}$. Other researchers are deploying microwaves in the name of 'green' chemistry, performing 
syntheses that usually require toxic or flammable solvents in just water, or even with no solvent at all ${ }^{3}$.

But in most cases it still takes a great deal of trial and error to optimize the conditions for microwave synthesis. "You have to put in the time to get the conditions right," Leadbeater says. "Too much power and you absolutely obliterate your materials; too little and nothing happens. But once you get it right, you're away." Indeed, after a set-up has been perfected, dozens of reactions can be run one after the other. The ability to halt reactions before they have the chance to produce contaminating by-products can also be important, and the new microwave reactors allow chemists to do this by cooling the tube containing the reactants with a blast of cold air.

Microwaves also have the useful property of heating some materials while leaving others cold - as anyone who has eaten a jam tart heated on full power for a minute in a domestic oven will testify. Essentially a highfrequency electric field, microwaves cause free ions or electrons to move in the same direction as the applied field. For polar molecules, such as water, which have an uneven distribution of electrical charge, the applied field forces them to wobble. Both of these types of movements generate heat. But other materials don't respond strongly to the field, and remain cool.

Inorganic chemists have taken advantage of this to make compounds such as metal sulphides, which have a variety of applications including as catalysts and metal coatings. Conventionally, the powdered metal and sulphur are simply mixed and heated in a sealed tube. But because the sulphur vaporizes and quickly increases the pressure, explosions are common - which means that the reaction must be carried
T he advantage of microwave chemistry is set to be a growth area.

out cautiously over several days. Microwaves heat only the metal, which then conducts the heat to the sulphur, allowing a less riskier reaction to take place. And - ping - the chemists get their metal sulphide in just 15 minutes (ref. 4).

Using conventional heat sources, the energy must first be conducted through the walls of the vessel containing the reactants. But microwaves heat the contents directly, allowing the temperature to rise much faster, and so boosting reaction rates. In fact, in many cases the increase in reaction rate is so great that researchers initially speculated that there was a strange effect at play, unrelated to the rapid supply of heat. This apparent 'microwave effect' puzzled chemists, some of whom invoked unconventional ideas such as microwaves causing a reduction in the amount of energy needed to start a reaction - to explain it.

\section{Supercharged}

Most experts now believe that the mysterious effect is the result of nothing more than microwaves' ability to superheat solvents way beyond their normal boiling points because the even spread of heat through the liquid allows it to reach a higher temperature before bubbles form. Water, for example, reaches $105^{\circ} \mathrm{C}$ before boiling in a microwave oven; whereas the solvent acetonitrile boils at $120^{\circ} \mathrm{C}$ instead of its usual $82^{\circ} \mathrm{C}$. "There are

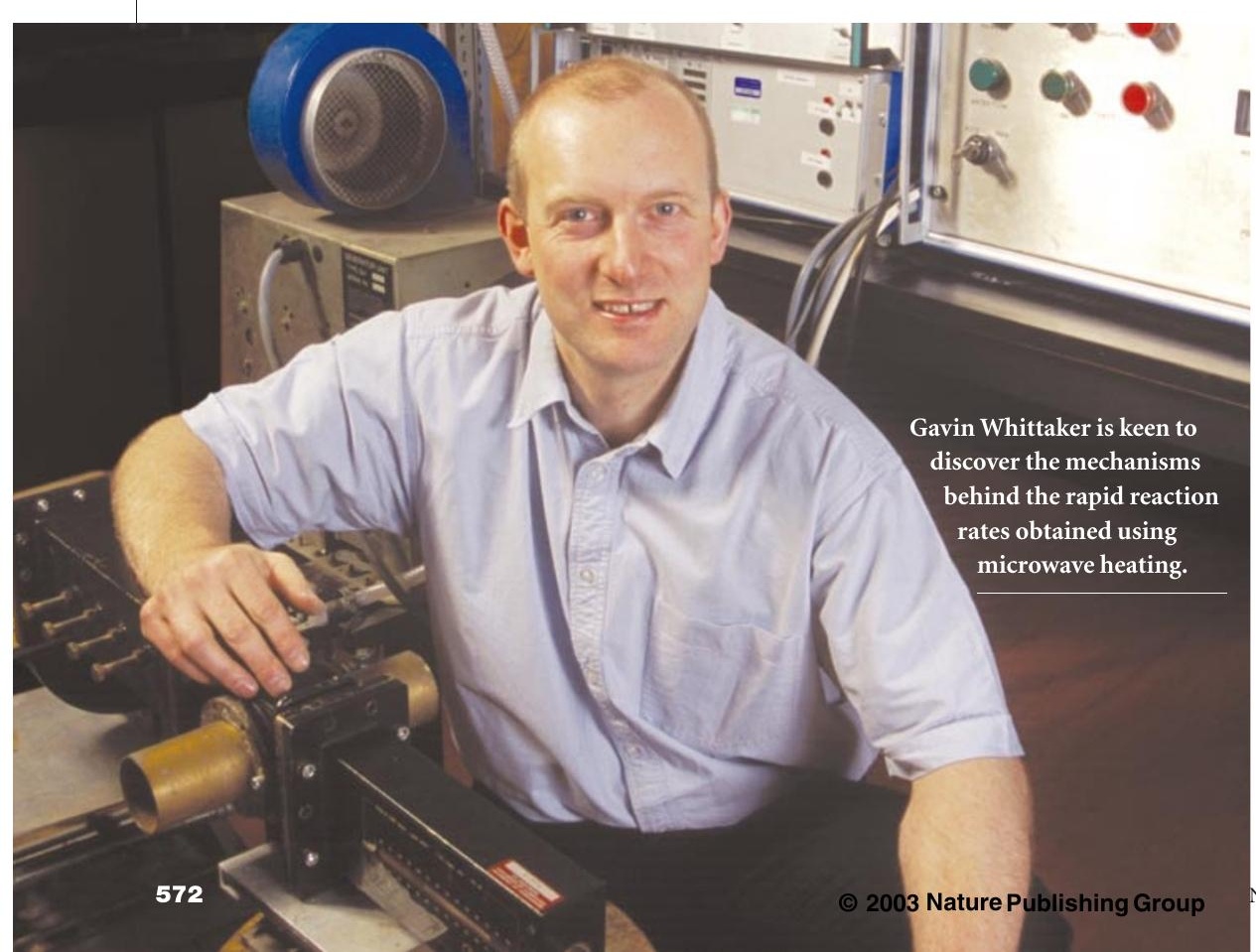

no general non-thermal microwave effects," asserts Kappe. "There is no magic."

Still, some scientists believe that questions about microwave mechanisms remain. Gavin Whittaker, a chemist at the University of Edinburgh, UK, who uses microwaves to prepare solid-state materials, is one. In unpublished work, he has observed "anomalies" in the microwave synthesis of barium titanate, used to make capacitors in the electronics industry. In microwave reactors, he says, the rate of reaction in many syntheses is so high it cannot be accounted for by the heating effects alone. "We've got evidence that there's an enhancement of diffusion of ions in the direction of the electric field," he claims.

Whittaker has presented his results at conferences, where some sceptics have suggested that they are an artefact caused by problems with the temperate measurements - which are notoriously difficult in solidstate systems. But Whittaker insists that the phenomenon is genuine. "It needs to be examined rigorously and properly, rather than people just saying it's down to bad measurements," he says. Other researchers have noted similar effects, most notably in the preparation of compounds such as aluminium oxide from powdered ingredients. John Booske and Reid Cooper, materials scientists at the University of WisconsinMadison, for example, argue that microwave heating increases the driving force for ionic diffusion when powdered ingredients are processed into ceramic materials ${ }^{5}$.

Whittaker believes that research into the mechanisms of microwave action is the most intriguing aspect of the field. "The exciting work at the moment is in understanding the fundamental principle of what happens," he argues. But even if such effects are found to be artefacts, and there are no new physical phenomena to explore, the simple advantage of speed means that microwave chemistry is poised to become a growth area.

"Industry has really caught on, but what's holding the academic field back now are the prices," says Kappe. The cheapest of the new generation of microwave reactors currently sells for about US\$20,000, beyond the buying power of many small academic labs. But Kappe is confident that market forces will soon rectify that situation. "As more and more academic chemists hear from their colleagues in industry about these devices, they will want to have one," he says. "And then competition between equipment suppliers will drive the prices down."

David Adam is a news and features writer for Nature.

1. Leadbeater, N. \& Marco, M. Angew. Chem. Int. Ed.

(in the press)

2. Stuerga, D., Gonon, K. \& Lallemant, M. Tetrahedron 49, 6229-6234 (1993).

. Varma, R. S. Pure Appl. Chem. 73, 193-198 (2001)

4. Whittaker, A. G. \& Mingos, D. M. P. J. Chem. Soc. Dalton Trans. 2073-2079 (1995)

5. Booske, J. H., Cooper, R. F., Freeman, S. A., Rybakov, K. I. \&

Semenov, V. E. Phys. Plasmas 5, 1664-1670 (1998). 